\title{
Scapular Kinematics in Athletes With and Without Rotator Cuff Tendinopathy: A Systematic Review
}

\author{
Xin Fu, Patrick Shu-hang Yung, Chun Cheong Ma, and Hio Teng Leong
}

\begin{abstract}
Context: Rotator cuff tendinopathy is one of the most frequently reported shoulder injuries in athletes of overhead sports. Abnormal scapular kinematics has been proposed as one of the contributing factors of rotator cuff tendinopathy in overhead athletes. Objectives: To review the literature on 3-dimensional scapular kinematics in overhead athletes with and without rotator cuff tendinopathy. Evidence Acquisition: Electronic databases (Cochrane Library, MEDLINE, Embase, and PubMed) were searched from inception to September 2017. In addition, the reference lists of the articles that met the inclusion criteria were also searched. We included studies that compared the changes in 3-dimensional scapular kinematics in athletes with and without rotator cuff tendinopathy. Two reviewers independently examined the quality of studies by using the modified Downs and Black checklist. Evidence Synthesis: A total of 9 studies (a total of 332 athletes, mean age 23.41 [2.62] y) were included in the final analysis. The methodological quality was low (modified Downs and Black checklist =9/15). Our findings showed a consistent pattern of increased scapular anterior tilting and internal rotation in the dominant shoulders than the nondominant shoulders of athletes who participated in overhead sports. Athletes of overhead sports seem to demonstrate an increase in scapular upward rotation during arm elevation when compared with nonathlete individuals. However, there is no consensus on the scapular kinematics pattern in athletes with rotator cuff tendinopathy when compared with healthy controls. Conclusion: Findings demonstrated that changes in scapular kinematics were observed in overhead athletes. However, all the included studies were cross-sectional studies with small sample size and diverse sports participation, whether changes in scapular kinematics may contribute to rotator cuff tendinopathy in overhead athletes warrants more high-quality prospective studies.
\end{abstract}

Keywords: overhead athletes, scapula, shoulder injury, biomechanics

The overhead throwing movement has been reported to be the fastest athletic movement performed in sports. ${ }^{1}$ During overhead throwing movement, high distraction forces place stress on the rotator cuff tendons and the supporting structures of the shoulder and result in shoulder pathologies. ${ }^{2}$ These repetitive, forceful, and quick overhead movements place athletes at high risk for shoulder injury. ${ }^{3}$ Rotator cuff tendinopathy is one of the most frequently reported shoulder injuries in overhead sports athletes, such as volleyball players $(23.7 \%)$, baseball pitchers $(71 \%)$, and swimmers (86\%-96\%). ${ }^{4-6}$ Rotator cuff tendinopathy is a commonly used clinical entity to encompass various shoulder pathologies, affecting the rotator cuff tendons and subacromial structures, such as rotator cuff-related pain, subacromial pain syndrome, subacromial bursitis, and shoulder impingement syndrome. ${ }^{7,8}$ It is characterized by pain, weakness, and impaired function and results in long periods of absence from training and competition. ${ }^{9}$ The etiology of rotator cuff tendinopathy in overhead athletes has been reported to be multifactorial, and a combination of extrinsic mechanical compression (ie, narrowing of the subacromial space) and tendon overuse/ overload (ie, repetitive overhead activities) has been proposed to be the major mechanism of rotator cuff tendinopathy. ${ }^{10}$

The coupled and coordinated movement between the scapula and humerus plays an important role for normal shoulder function, particularly in overhead athletes. ${ }^{11}$ The scapular motion during active humeral elevation in healthy individuals has been defined as a pattern of progressive scapular upward rotation, posterior tilting,

The authors are with the Department of Orthopaedics and Traumatology, The Chinese University of Hong Kong, Shatin, Hong Kong. Leong (annieleonght@ cuhk.edu.hk) is corresponding author. and high variable internal/external rotation in relation to the thorax. ${ }^{12-15}$ According to Ludewig and Reynolds, ${ }^{13}$ scapular upward rotation is the primary scapular motion and is important for the elevation of the lateral acromion to preserve the subacromial space during arm elevation and to prevent rotator cuff compression. Scapular posterior tilting and scapular external rotation are considered as a secondary scapular motion and an accessory motion of the scapula, respectively, and both motions move the anterior acromion posteriorly to avoid rotator cuff compression. Previous studies have reported changes in scapular kinematics between the dominant and nondominant shoulders of athletes who were involved in overhead sports ${ }^{16,17}$ and between athletes of overhead sports and nonathlete controls ${ }^{17,18}$ and were believed to be a sportspecific adaption in overhead athletes. ${ }^{18,19}$ Nevertheless, altered scapular kinematics was also identified in overhead athletes with rotator cuff tendinopathy ${ }^{19-22}$ and has been proposed as one of the contributing factors for rotator cuff tendinopathy. ${ }^{19,23,24}$

Several systematic reviews on changes in scapular kinematics have been carried out in specific shoulder pathologies such as subacromial impingement syndrome and different shoulder musculoskeletal disorders ${ }^{25,26}$; however, no systematic review summarized the results of studies on the changes in scapular kinematics in overhead athletes with and without rotator cuff tendinopathy. In view of the current uncertainty regarding the changes in scapular kinematics in athletes of overhead sports, the aim of this study is to systematically review the literature and to summarize the scapular kinematics patterns in overhead athletes with and without rotator cuff tendinopathy. Finding from this study may provide a better understanding of the pathogenesis of rotator cuff tendinopathy in athletes of overhead sports and underpin preventive and rehabilitative programs. 


\section{Methods}

Published articles that examined the scapular kinematics in overhead athletes with and without rotator cuff tendinopathy were reviewed using the guidelines by the Preferred Reporting Items for Systematic Reviews and Meta-Analyses statement. ${ }^{27}$ Our review has been registered with PROSPERO database (registration number: CRD42017069708).

\section{Search Strategy}

An electronic database search was performed using Cochrane Library, MEDLINE, Embase, and PubMed from inception to September 2017. The search strategy was presented in Table 1. Supplementary searches were carried out by hand searching the reference lists of the included studies. Articles were imported into reference manager software (EndNote X7; Thomson Reuters, New York, NY) to remove duplicates. One reviewer (X.F.) screened all titles and/or abstracts for relevance and duplication. Relevant studies were accessed for full-text review prior to inclusion in the systematic review.

\section{Study Inclusion and Exclusion Criteria}

Studies were included if (1) athletes who involved in overhead sports (including baseball, volleyball, tennis, softball, water polo, handball, swimming, field events, badminton, basketball, squash, and racquetball, etc) ${ }^{28}$; (2) overhead athletes presented with signs and symptoms suggestive of rotator cuff tendinopathy/tendinitis/ tendinosis, shoulder impingement syndrome, or subacromial bursitis diagnosed by clinical tests and/or conventional imaging ${ }^{29-31}$;

\section{Table 1 Search Term}

\begin{tabular}{l} 
Search term \\
1. Rotator cuff.mp. \\
2. Rotator cuff tendon.mp. \\
3. Subscapularis.mp. \\
4. Supraspinatus.mp. \\
5. Infraspinatus.mp. \\
6. Teres minor.mp. \\
7. Subacromial bursa.mp. \\
8. 1 or 2 or 3 or 4 or 5 or 6 or 7 \\
9. Tendinopathy.mp. \\
10. Tendinitis.mp. \\
11. Tendinosis.mp. \\
12. Shoulder impingement. mp. \\
13. Subacromial impingement.mp. \\
14. Suacromial bursitis.mp. \\
15. 9 or 10 or 11 or 12 or 13 or 14 \\
16. Sport \\
17. Athlete* \\
18. Player* \\
19. 16 or 17 or 18 \\
20. Scapula*.mp. \\
21. 9 AND 15 AND 19 AND 20 \\
22. 21 limited to English language, full text, human studies \\
\hline
\end{tabular}

(3) use of a motion analyzer or similar kinematic methods to calculate scapular kinematics defined as scapular upward/downward rotation, scapular anterior/posterior tilting, and scapular internal/external rotation; and (4) the study report was published in English and full-text articles prior to September 2017. Studies were excluded if (1) participants were diagnosed with rotator cuff full thickness tear or calcified tendinopathy and other postsurgical condition $^{29-31}$; (2) the study population involved animal models or cadavers; and (3) the study report was published as narrative reviews, editorials, commentaries, and opinion-based papers.

\section{Assessment of Methodological Quality and Risk of Bias}

The methodological quality of each study was assessed independently by 2 reviewers (X.F. and C.C.M.) using the modified Downs and Black checklist. The Downs and Black checklist was modified to include criteria that were relevant to assess potential bias in the included studies, with a maximum score of $15 .{ }^{32,33}$ A total score of $\geq 12$ indicates high methodological quality, a score of 10 or 11 indicates moderate quality, whereas a score $\leq 9$ indicates low quality. ${ }^{32}$ Disagreements in scores were resolved by consensus between the 2 reviewers or by a third opinion (H.T.L.) when required. Intraclass correlation coefficient (ICC) 2-way mixedeffects analysis was calculated using SPSS for Windows (version 24; SPSS Inc, Chicago, IL) to measure the interrater reliability between the 2 reviewers for quality assessment.

\section{Data Extraction and Synthesis}

All data were extracted by 2 reviewers (X.F. and H.T.L.). Data extraction was based on a standardized form that includes (1) the characteristics of the study (authors and years); (2) the characteristics of participants (sample size, study population, gender, and age); (3) diagnosis criteria of rotator cuff tendinopathy; (4) method used to measure scapular kinematics and its reliability; and (5) reported significant results published in mean and SD on the changes in scapular upward/downward rotation, scapular anterior/posterior tilting, and scapular internal/external rotation.

\section{Results}

\section{Study Selection}

The electronic search identified 684 relevant studies, and 4 additional papers were identified by hand searching from the references lists of the studies. After excluding 281 duplicates, we screened 407 titles/abstracts, and 386 irrelevant articles were excluded. The remaining 21 articles were obtained for full-text review, of which 9 articles met the inclusion criteria and were included in this systematic review (Figure 1).

\section{Study Characteristics}

A total of 332 participants $($ men $=331$ and women $=21)$ with a mean age of 23.41 (2.62) years were included into the review. The sample size ranged from 21 to 60 , and the age of the participants ranged between 18 and 32 years. Wide range of overhead athletes, such as baseball, ${ }^{16-19,21}$ volleyball, ${ }^{17,20,34,35}$ handball, ${ }^{34,35}$ swimming, ${ }^{22}$ and tennis, ${ }^{17}$ were examined. Of the included studies, 4 studies compared the changes between the dominant (throwing) and nondominant shoulders of athletes who 


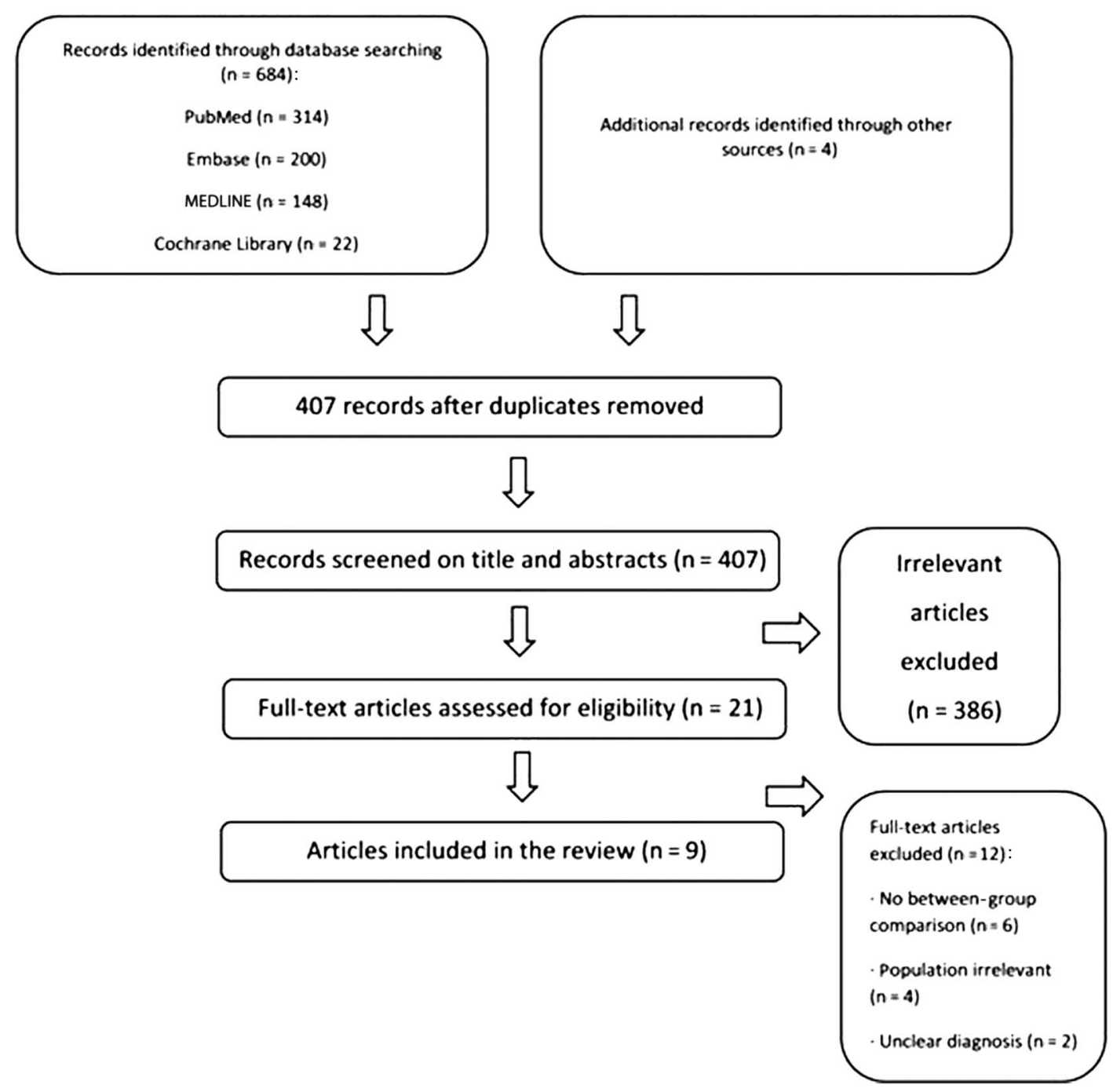

Figure 1 - Flow diagram of the search strategy.

were involved in overhead sports (Table 2), ${ }^{16,17} 3$ studies compared the scapular kinematics between athletes of overhead sports and nonathlete controls (Table 3), ${ }^{18,34,35}$ and the remaining 4 studies compared the scapular kinematics between athletes with and without rotator cuff tendinopathy (Table 4). ${ }^{19-22}$ Clinical tests (Neer test and Hawkins test) were performed in 2 studies to determine the diagnosis of rotator cuff tendinopathy: ${ }^{21,22}$ one study used magnetic resonance imaging ${ }^{19}$ and one study combined the use of clinical tests (painful arc, resisted external rotation, Jobe test, Neer test, and Hawkins test) and ultrasound imaging to confirm the diagnosis of rotator cuff tendinopathy (Table 4). ${ }^{20}$

\section{Quality and Level of Evidence}

The level of evidence of all the included studies was classified as level 3 (ie, cross-sectional design). The methodological quality scores of included articles were reported in Table 5. On the basis of the modified Downs and Black criteria, the median methodological quality of all 11 studies was $9 / 15$. The study quality of all included studies was low. The ICC for the interrater reliability between the 2 reviewers was .75 (95\% confidence interval, .03-.94).

\section{Scapular Kinematics Measurement Protocol}

The majority of the studies used an electromagnetic tracking device to examine the scapular kinematics during humeral elevation at the scapular plane, ${ }^{17-19,21,35}$ and the reliability results were reported to be moderate to excellent (ICC ranging from .61 to $.99,{ }^{17-20,35}$ and the standard error of measurement was $\left.0.3^{\circ}\right) .{ }^{17}$ Leong et al ${ }^{20}$ used the optical-based motion analysis system to capture the scapular kinematics during dynamic shoulder abduction from $0^{\circ}$ to $90^{\circ}$, and the reliability results were reported to be good to excellent (ICC ranging from .71 to .90 , minimal detectable changes ranged from $1.3^{\circ}$ to $\left.3.7^{\circ}\right) .{ }^{20}$ Other studies used a digital inclinometer to measure the changes in scapular upward rotation during static humeral elevation at the scapular plane ${ }^{16,22}$ and frontal plane, ${ }^{34}$ and the reliability results were reported to be excellent (ICC ranging from .86 to .99$).{ }^{16,22,34}$

\section{Scapular Kinematics in Healthy Overhead Athletes}

Of the 4 studies that compared the changes in scapular kinematics between the dominant and nondominant shoulders of athletes who were involved in overhead sports, ${ }^{16,17} 2$ out of 4 studies reported 


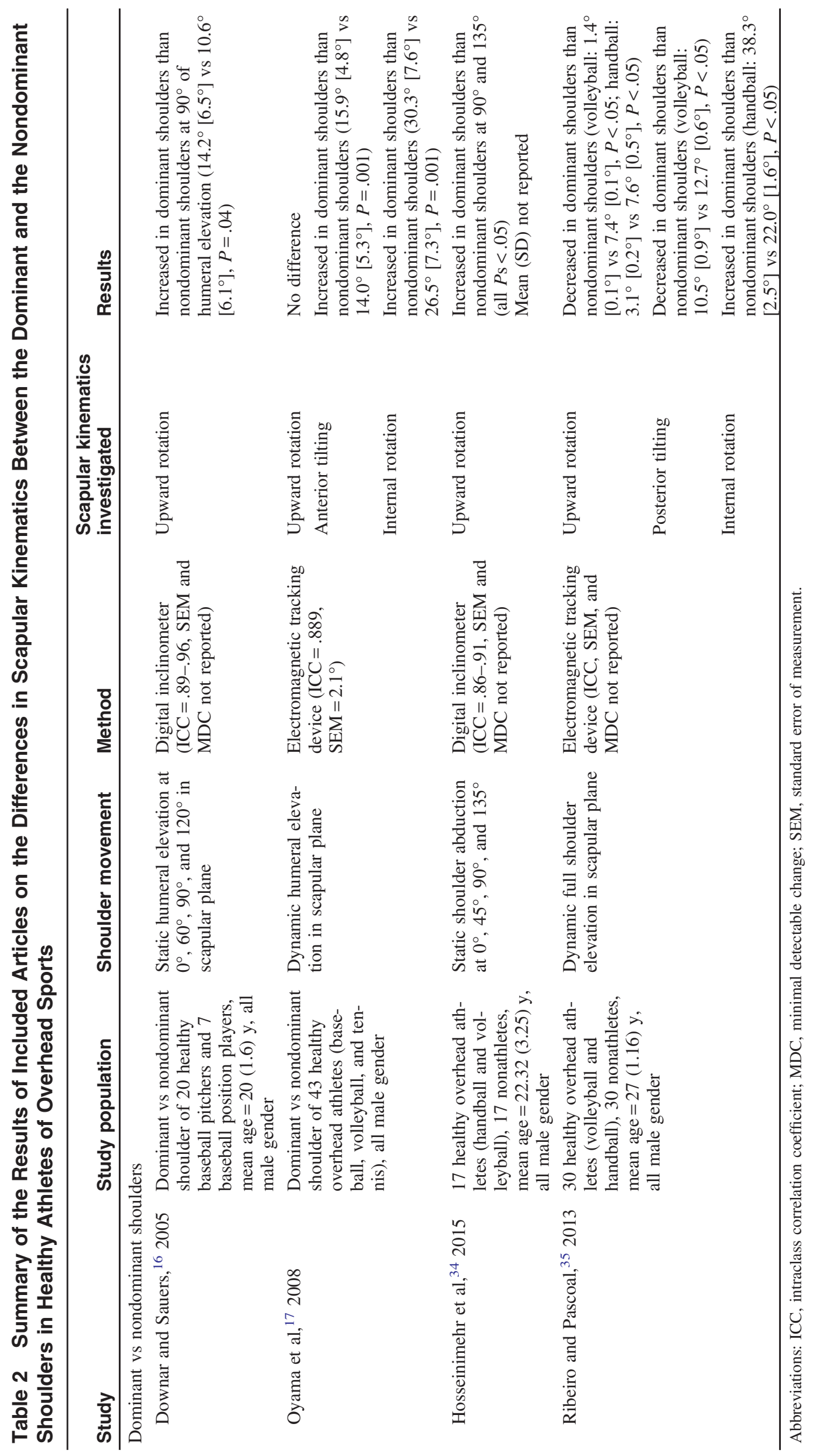




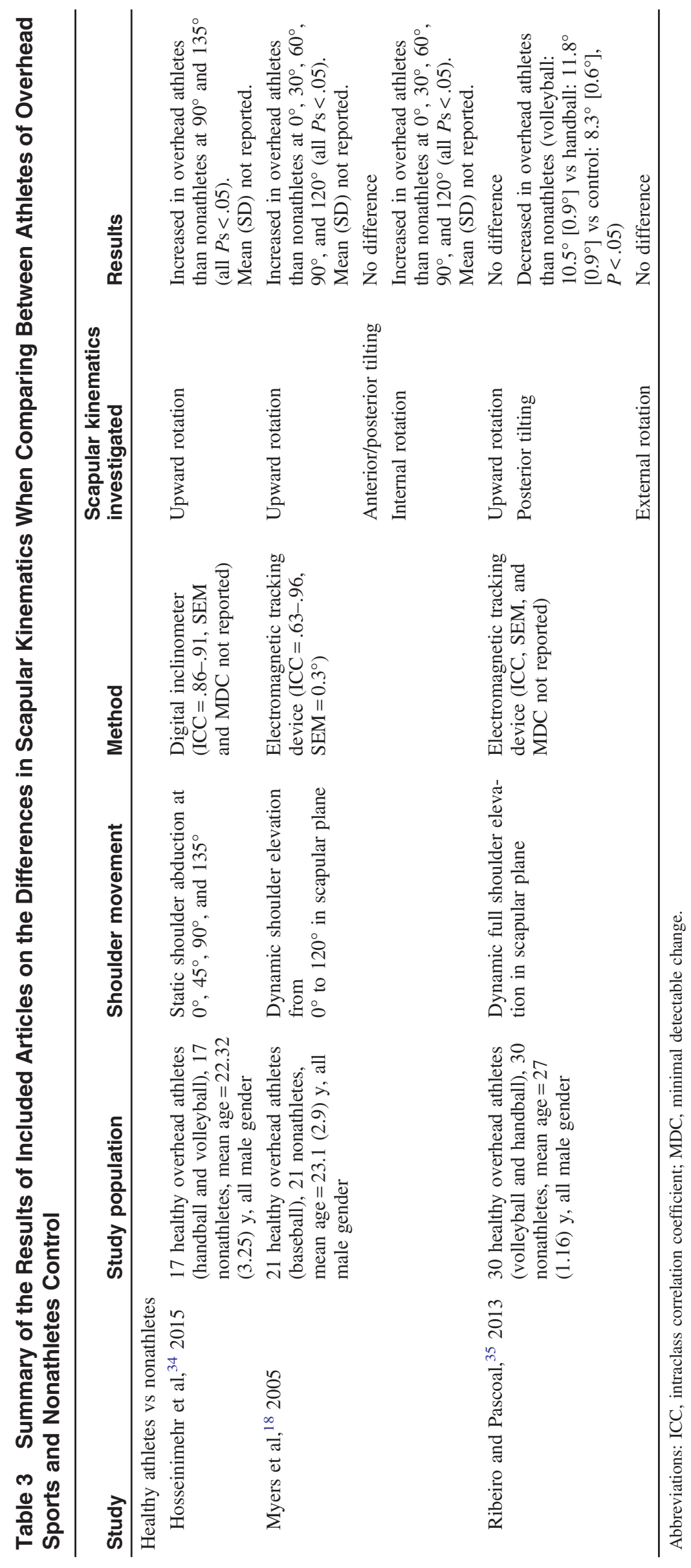




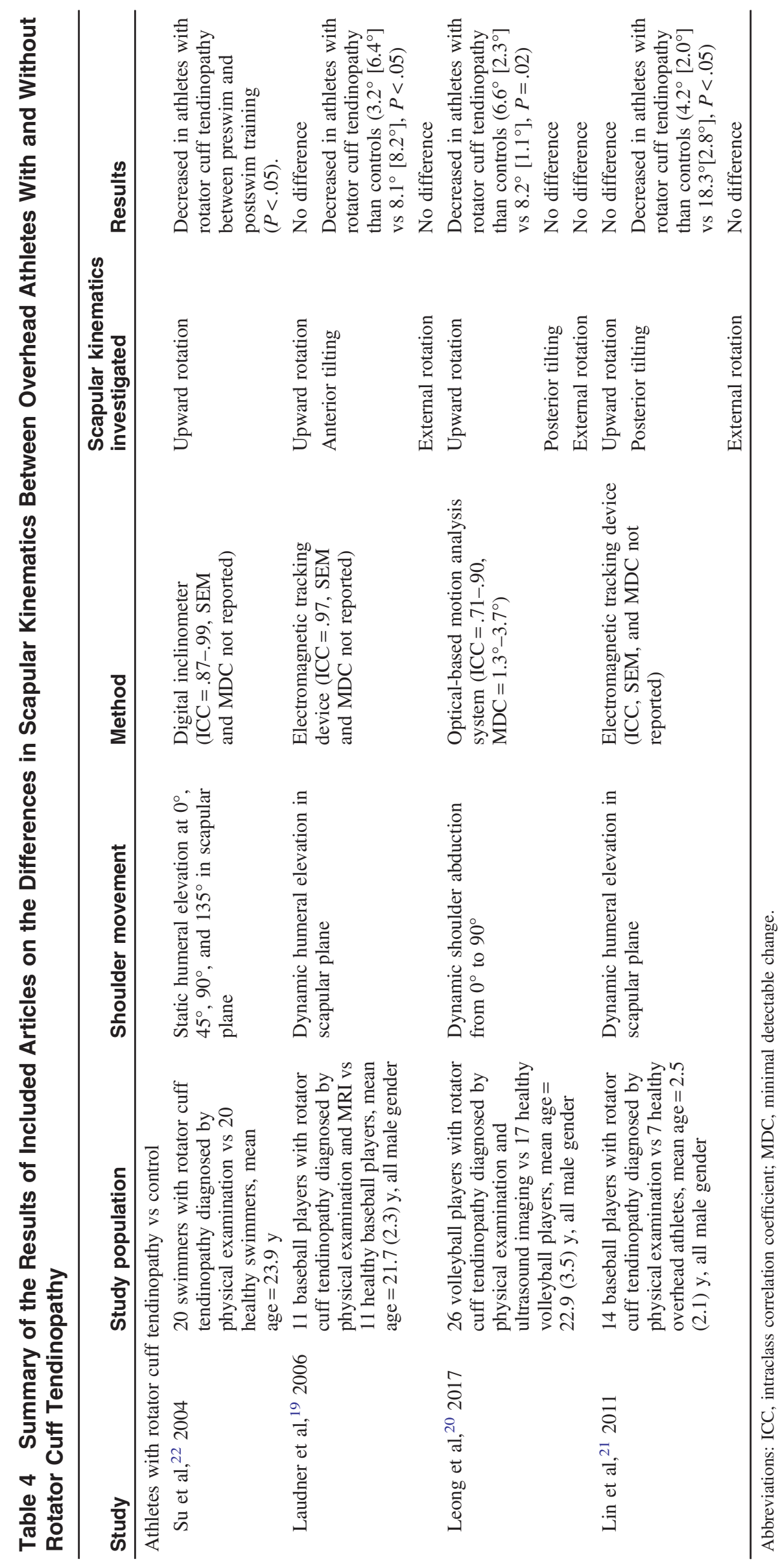


Table 5 Methodological Quality Assessment and Level of Evidence

\begin{tabular}{|c|c|c|c|c|c|c|c|c|c|c|c|c|c|c|c|c|c|}
\hline \multicolumn{17}{|c|}{ Downs and Black checklist $^{a}$ items included $^{b}$} & \multirow{2}{*}{$\frac{\text { Level of evidence }^{c}}{3}$} \\
\hline Study & 1 & 2 & 3 & 5 & 6 & 7 & 10 & 11 & 12 & 16 & 18 & 20 & 21 & 22 & 25 & Total & \\
\hline Downar and Sauers ${ }^{16}$ & 1 & 1 & 1 & 0 & 1 & 1 & 1 & 0 & 0 & 1 & 1 & 1 & 1 & 0 & 0 & 10 & 3 \\
\hline Hosseinimehr et al ${ }^{34}$ & 1 & 1 & 1 & 0 & 1 & 1 & 1 & 0 & 0 & 1 & 1 & 1 & 0 & 0 & 0 & 9 & 3 \\
\hline Laudner et al ${ }^{19}$ & 1 & 1 & 1 & 0 & 1 & 1 & 1 & 0 & 0 & 1 & 1 & 1 & 0 & 0 & 0 & 9 & 3 \\
\hline Leong et $\mathrm{al}^{20}$ & 1 & 1 & 1 & 0 & 1 & 1 & 1 & 0 & 0 & 1 & 1 & 1 & 0 & 0 & 0 & 9 & 3 \\
\hline Lin et $\mathrm{al}^{21}$ & 1 & 1 & 1 & 0 & 1 & 1 & 1 & 0 & 0 & 1 & 1 & 1 & 0 & 0 & 0 & 9 & 3 \\
\hline Myers et al ${ }^{18}$ & 1 & 1 & 1 & 0 & 1 & 1 & 1 & 0 & 0 & 1 & 1 & 1 & 0 & 0 & 0 & 9 & 3 \\
\hline Ribeiro and Pascoal ${ }^{35}$ & 1 & 1 & 1 & 0 & 1 & 1 & 1 & 0 & 0 & 1 & 1 & 0 & 0 & 0 & 0 & 8 & 3 \\
\hline Oyama et al ${ }^{17}$ & 1 & 1 & 1 & 0 & 1 & 1 & 1 & 0 & 0 & 1 & 1 & 1 & 0 & 0 & 0 & 9 & 3 \\
\hline Su et $\mathrm{al}^{22}$ & 1 & 1 & 1 & 0 & 1 & 1 & 1 & 0 & 0 & 1 & 1 & 1 & 0 & 0 & 0 & 9 & 3 \\
\hline
\end{tabular}

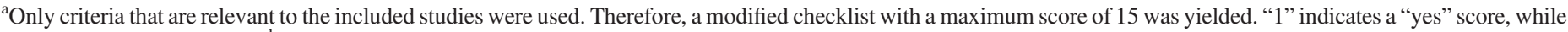

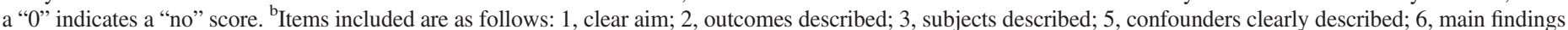

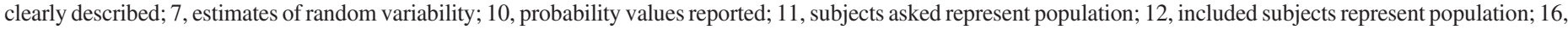

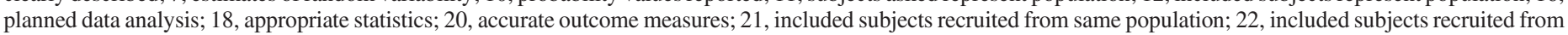
same period of time; 25, adjustment for confounding in analyses. ${ }^{\mathrm{c}}$ Oxford Centre for Evidence-Based Medicine.

significant increase in scapular upward rotation, ${ }^{16}$ whereas one study reported significant decrease in upward rotation in dominant shoulders than in nondominant shoulders, ${ }^{35}$ and one study found no difference when comparing between the dominant and the nondominant shoulders. ${ }^{17}$ In addition, both studies from Oyama et al ${ }^{17}$ and Ribeiro and Pascoal ${ }^{35}$ demonstrated a significant increase in scapular anterior tilting and internal rotation in the dominant shoulders than the nondominant shoulders of overhead athletes (Table 2). ${ }^{17,35}$

Of the 3 studies that compared the changes in scapular kinematics between overhead athletes and nonathlete individuals, ${ }^{18,34,35} 2$ out of 3 studies reported a significant increase in scapular upward rotation in overhead athletes than in nonathletes control, ${ }^{18,34}$ whereas the other study reported no difference in scapular upward rotation. ${ }^{35}$ However, inconsistent findings were reported in the scapular internal-external rotation and anteriorposterior tilting between athletes and nonathletes group (Table 3). ${ }^{18,34,35}$

\section{Scapular Kinematics in Overhead Athletes With Rotator Cuff Tendinopathy}

Among the 4 studies that compared the scapular kinematics in athletes with and without rotator cuff tendinopathy, 2 out of 4 studies reported a significant decrease in scapular upward rotation in athletes with rotator cuff tendinopathy, ${ }^{20,22}$ whereas the remaining 2 studies found no significant difference. ${ }^{19,21}$, All studies showed no difference in the scapular external rotation when comparing athletes with and without rotator cuff tendinopathy. ${ }^{19-21}$ However, conflicting results were reported in the change in scapular posterior tilting in athletes with rotator cuff tendinopathy (Table 4). ${ }^{19-21}$

\section{Discussion}

This systematic review summarized the changes in scapular kinematics in asymptomatic overhead athletes and those with rotator cuff tendinopathy. All the included studies were classified as level 3 (ie, cross-sectional design). From our systematic review, consistent pattern of increased scapular anterior tilting and internal rotation was reported in the dominant shoulders than the nondominant shoulders of athletes who participated in overhead sports. Athletes of overhead sports seem to demonstrate an increase in scapular upward rotation during arm elevation when compared with nonathlete individuals. However, there is no consensus on the scapular kinematics pattern in athletes with rotator cuff tendinopathy when compared with healthy controls.

\section{Scapular Kinematics in Healthy Overhead Athletes}

From our systematic review, a consistent pattern of increased scapular anterior tilting and internal rotation was reported in the dominant shoulders than in the nondominant shoulders of athletes who participated in overhead sports. Previous studies have shown significant loss of internal rotation range of motion (glenohumeral internal rotation deficit), ${ }^{36}$ increased humeral retroversion, ${ }^{37,38}$ tightness of the posterior shoulder capsule and muscles, ${ }^{39,40}$ and tightness of pectoralis minor ${ }^{41,42}$ in the dominant shoulders of athletes who participated in overhead sports, and these were associated with increased scapular anterior tilting and internal rotation. ${ }^{43-45}$ In this connection, the glenohumeral contact pressure was significantly increased with more scapular internal rotation in a cadaveric study. ${ }^{46}$ In this way, athletes of overhead sports may be more vulnerable to rotator cuff tendinopathy as these changes are believed to irritate the rotator cuff tendons and the subacromial tissues when the acromial failed to elevation during arm elevation. ${ }^{13}$

On the other hand, athletes of overhead sports seem to demonstrate an increase in scapular upward rotation during arm elevation when compared with nonathlete individuals. ${ }^{16,18,34}$ This increase in scapular upward rotation during arm elevation has been suggested as a chronic adaptation to preserve the subacromial space and to prevent rotator cuff compression during the throwing motion. ${ }^{12,18,47}$

\section{Scapular Kinematics in Overhead Athletes With Rotator Cuff Tendinopathy}

Decreased scapular upward rotation and increased anterior tilting and internal rotation during arm elevation were believed to contribute to rotation cuff tendinopathy when the acromial failed to achieve adequate clearance of the rotator cuff tendons during arm elevation $^{48}$; however, our current systematic review did not support 
this assumption. Among the 4 studies that compared the scapular kinematics in athletes with and without rotator cuff tendinopathy, conflicting results were reported, and there is no consensus on the scapular kinematics pattern in athletes with rotator cuff tendinopathy when compared with healthy controls. Weakness or imbalanced activation of the scapular muscles has been reported in athletes with rotator cuff tendinopathy, ${ }^{20,21,23,49}$ and these were associated with alterations in scapular kinematics ${ }^{20,21,23}$ and narrowing of the subacromial space. ${ }^{49}$ Strength deficits of the lower trapezius and serratus anterior have been reported to be moderately associated with decreased scapular upward rotation in overhead athletes with clinically identified scapular dyskinesis. ${ }^{48}$ Lin et al ${ }^{21}$ also demonstrated the decrease in electromyography activity of the serratus anterior to be correlated with the decrease in posterior tilting of the scapula in overhead athletes with rotator cuff tendinopathy. In addition, Leong et $\mathrm{al}^{47}$ reported that delayed activity onset of middle and lower trapezius relative to upper trapezius was associated with decreased scapular rotation in athletes with rotator cuff tendinopathy. ${ }^{20}$ More high-quality prospective studies are required to investigate the changes in scapular kinematics in overhead athletes with rotator cuff tendinopathy for the prevention and management of rotator cuff tendinopathy.

\section{Clinical Implications and Future Directions}

Changes in scapular kinematics may be an adaptation to sports practice in athletes of overhead sports; however, the exact mechanism on how these changes may be related to shoulder disorders remains unknown. Whether changes in scapular kinematics may contribute to rotator cuff tendinopathy warrants further investigation with high-quality prospective study. There is no consensus on the scapular kinematics pattern in athletes with rotator cuff tendinopathy when compared with healthy controls. Scapular-focused intervention has been developed to address scapular muscle deficits in patients with rotator cuff tendinopathy. ${ }^{50,51}$ Future studies should investigate whether appropriate scapular-focused intervention would rectify the maladaptation of scapular kinematics in athletes with rotator cuff tendinopathy.

\section{Limitations}

Several limitations that need to be considered in this study are as follows: (1) All the included studies were cross-sectional with small sample size and diverse sports participation. More prospective studies are required to identify the changes in scapular kinematics in athletes of overhead sports and how it may contribute to rotator cuff tendinopathy. (2) Caution should be taken when interpreting the findings due to the variation on the methodology in capturing the 3-dimensional scapular kinematics. Different motion capture systems and Euler decomposition as well as different arm elevation task might affect the results of the included studies. ${ }^{25,52}$ (3) Publication bias existed because we excluded non-English articles, unpublished articles, and English studies without access. (4) Meta-analysis was impossible due to the small number of studies, and the studies were largely heterogeneous.

\section{Conclusion}

From our systematic review, increased scapular anterior tilting and internal rotation were reported in the dominant shoulders than in the nondominant shoulders of athletes who participated in overhead sports. Athletes of overhead sports seem to demonstrate an increase in scapular upward rotation during arm elevation when compared with nonathlete individuals. However, there is no consensus on the scapular kinematics pattern in athletes with rotator cuff tendinopathy when compared with healthy controls. More high-quality studies are required to identify the scapular kinematic patterns in athletes with and without rotator cuff tendinopathy.

\section{Acknowledgments}

This article is an original research in sports injury and rehabilitation being submitted to Journal of Sport Rehabilitation online. The authors hereby declare that there is no external financial support for this study. They also declare that this article deals with original material that has not been submitted or published elsewhere.

\section{References}

1. Dugas JR, Mathis TP. Partial rotator cuff tears in throwing athletes. Oper Tech Sports Med. 2016;24:196-202. doi:10.1053/j.otsm.2016. 04.007

2. Fleisig GS, Andrews JR, Dillman CJ, Escamilla RF. Kinetics of baseball pitching with implications about injury mechanisms. Am J Sports Med. 1995;23:233-239. doi:10.1177/036354659502300218

3. Wilk KE, Obma P, Simpson CD, Cain EL, Dugas JR, Andrews JR. Shoulder injuries in the overhead athlete. J Orthop Sports Phys Ther. 2009;39:38-54. doi:10.2519/jospt.2009.2929

4. McFarland EG, Wasik M. Epidemiology of collegiate baseball injuries. Clin J Sport Med. 1998;8:10-13. PubMed ID: 9448950 doi:10. 1097/00042752-199801000-00003

5. Rodeo SA, Nguyen JT, Cavanaugh JT, Patel Y, Adler RS. Clinical and ultrasonographic evaluations of the shoulders of elite swimmers. Am J Sports Med. 2016;44:3214-3221. doi:10.1177/0363546 516657823

6. Wang H, Cochrane T. A descriptive epidemiological study of shoulder injury in top level English male volleyball players. Int J Sports Med. 2001;22:159-163. PubMed ID: 11281621 doi:10.1055/s-200111346

7. Dunn WR, Kuhn JE, Sanders R, et al. Symptoms of pain do not correlate with rotator cuff tear severity: a cross-sectional study of 393 patients with a symptomatic atraumatic full-thickness rotator cuff tear. J Bone Joint Surg Am. 2014;96:793-800. PubMed ID: 24875019 doi:10.2106/JBJS.L.01304

8. Harrison AK, Flatow EL. Subacromial impingement syndrome. J Am Acad Orthop Surg. 2011;19:701-708. PubMed ID: 22052646 doi:10. 5435/00124635-201111000-00006

9. Verhagen EA, Van der Beek AJ, Bouter LM, Bahr RM, Van Mechelen W. A one season prospective cohort study of volleyball injuries. Br J Sports Med. 2004;38:477-481. PubMed ID: 15273190 doi:10.1136/bjsm.2003.005785

10. Soslowsky LJ, Thomopoulos S, Esmail A, et al. Rotator cuff tendinosis in an animal model: role of extrinsic and overuse factors. Ann Biomed Eng. 2002;30:1057-1063. PubMed ID: 12449766 doi:10. 1114/1.1509765

11. Kibler BW, Sciascia A, Wilkes T. Scapular dyskinesis and its relation to shoulder injury. J Am Acad Orthop Surg. 2012;20:364-372. PubMed ID: 22661566 doi:10.5435/JAAOS-20-06-364

12. Kibler WB. The role of the scapula in athletic shoulder function. Am J Sports Med. 1998;26:325-337. PubMed ID: 9548131 doi:10.1177/ 03635465980260022801

13. Ludewig PM, Reynolds JF. The association of scapular kinematics and glenohumeral joint pathologies. J Orthop Sports Phys Ther. 
2009;39:90-104. PubMed ID: 19194022 doi:10.2519/jospt.2009. 2808

14. McClure PW, Michener LA, Karduna AR. Shoulder function and 3dimensional scapular kinematics in people with and without shoulder impingement syndrome. Phys Ther. 2006;86:1075-1090. PubMed ID: 16879042

15. McQuade KJ, Borstad J, de Oliveira AS. Critical and theoretical perspective on scapular stabilization: what does it really mean, and are we on the right track? Phys Ther. 2016;96:1162-1169. PubMed ID: 26847012 doi: $10.2522 / \mathrm{ptj} .20140230$

16. Downar JM, Sauers EL. Clinical measures of shoulder mobility in the professional baseball player. J Athl Train. 2005;40:23-29. PubMed ID: 15902320

17. Oyama S, Myers JB, Wassinger CA, Daniel Ricci R, Lephart SM. Asymmetric resting scapular posture in healthy overhead athletes. J Athl Train. 2008;43:565-570. PubMed ID: 19030133 doi:10.4085/ 1062-6050-43.6.565

18. Myers JB, Laudner KG, Pasquale MR, Bradley JP, Lephart SM. Scapular position and orientation in throwing athletes. Am J Sports Med. 2005;33:263-271. doi:10.1177/0363546504268138

19. Laudner KG, Myers JB, Pasquale MR, Bradley JP, Lephart SM. Scapular dysfunction in throwers with pathologic internal impingement. J Orthop Sports Phys Ther. 2006;36:485-494. doi:10.2519/ jospt.2006.2146

20. Leong HT, Ng GY, Chan SC, Fu SN. Rotator cuff tendinopathy alters the muscle activity onset and kinematics of scapula. J Electromyogr Kinesiol. 2017;35:40-46. PubMed ID: 28595162 doi:10.1016/j. jelekin.2017.05.009

21. Lin JJ, Hsieh SC, Cheng WC, Chen WC, Lai Y. Adaptive patterns of movement during arm elevation test in patients with shoulder impingement syndrome. J Orthop Res. 2011;29:653-657. PubMed ID: 21437944 doi:10.1002/jor.21300

22. Su KPE, Johnson MP, Gracely EJ, Karduna AR. Scapular rotation in swimmers with and without impingement syndrome: practice effects. Med Sci Sports Exerc. 2004;36:1117-1123. PubMed ID: 15235314 doi:10.1249/01.MSS.0000131955.55786.1A

23. Ludewig PM, Cook TM. Alterations in shoulder kinematics and associated muscle activity in people with symptoms of shoulder impingement. Phys Ther. 2000;80:276-291. PubMed ID: 10696154

24. Phadke V, Camargo P, Ludewig P. Scapular and rotator cuff muscle activity during arm elevation: a review of normal function and alterations with shoulder impingement. Rev Bras Fisioter. 2009;13:1-9. PubMed ID: 20411160 doi:10.1590/S1413-355520 09005000012

25. Keshavarz R, Bashardoust Tajali S, Mir SM, Ashrafi H. The role of scapular kinematics in patients with different shoulder musculoskeletal disorders: a systematic review approach. J Bodyw Mov Ther. 2017;21:386-400. PubMed ID: 28532884 doi:10.1016/j.jbmt.2016. 09.002

26. Timmons MK, Thigpen CA, Seitz AL, Karduna AR, Arnold BL, Michener LA. Scapular kinematics and subacromial-impingement syndrome: a meta-analysis. J Sport Rehabil. 2012;21:354-370. PubMed ID: 22388171 doi:10.1123/jsr.21.4.354

27. Moher D, Liberati A, Tetzlaff J, Altman DG; PRISMA Group. Preferred reporting items for systematic reviews and meta-analyses: the PRISMA statement. Ann Intern Med. 2009;151:264-269. PubMed ID: 19622511 doi:10.7326/0003-4819-151-4-20090818000135

28. Burn MB, McCulloch PC, Lintner DM, Liberman SR, Harris JD. Prevalence of scapular dyskinesis in overhead and nonoverhead athletes: a systematic review. Orthop J Sports Med. 2016; 4:2325967115627608. doi:10.1177/2325967115627608.
29. Desjardins-Charbonneau A, Roy JS, Dionne CE, et al. The efficacy of manual therapy for rotator cuff tendinopathy: a systematic review and meta-analysis. J Orthop Sports Phys Ther. 2015;45:330-350. PubMed ID: 25808530 doi:10.2519/jospt.2015.5455

30. Desmeules F, Boudreault J, Dionne CE, et al. Efficacy of exercise therapy in workers with rotator cuff tendinopathy: a systematic review. J Occup Health. 2016;58:389-403. PubMed ID: 27488037 doi:10.1539/joh.15-0103-RA

31. Toliopoulos P, Desmeules F, Boudreault J, et al. Efficacy of surgery for rotator cuff tendinopathy: a systematic review. Clin Rheumatol. 2014;33:1373-1383. PubMed ID: 24682606 doi:10.1007/s10067014-2563-9

32. Hart HF, Culvenor AG, Collins NJ, et al. Knee kinematics and joint moments during gait following anterior cruciate ligament reconstruction: a systematic review and meta-analysis. Br J Sports Med. 2016; 50:597-612. PubMed ID: 26265562 doi:10.1136/bjsports-2015094797

33. Irving DB, Cook JL, Menz HB. Factors associated with chronic plantar heel pain: a systematic review. J Sci Med Sport. 2006;9:1122. PubMed ID: 16584917 doi:10.1016/j.jsams.2006.02.004

34. Hosseinimehr SH, Anbarian M, Norasteh AA, Fardmal J, Khosravi MT. The comparison of scapular upward rotation and scapulohumeral rhythm between dominant and non-dominant shoulder in male overhead athletes and non-athletes. Man Ther. 2015;20:758-762. PubMed ID: 25795109 doi:10.1016/j.math.2015.02.010

35. Ribeiro A, Pascoal AG. Resting scapular posture in healthy overhead throwing athletes. Man Ther. 2013;18:547-550. PubMed ID: 23791560 doi:10.1016/j.math.2013.05.010

36. Borsa PA, Dover GC, Wilk KE, Reinold MM. Glenohumeral range of motion and stiffness in professional baseball pitchers. Med Sci Sports Exer. 2006;38:21-26. doi:10.1249/01.mss.0000180890.69932.15

37. Osbahr DC, Cannon DL, Speer KP. Retroversion of the humerus in the throwing shoulder of college baseball pitchers. Am J Sports Med. 2002;30:347-353. PubMed ID: 12016074 doi:10.1177/036354 65020300030801

38. Reagan KM, Meister K, Horodyski MB, Werner DW, Carruthers C, Wilk K. Humeral retroversion and its relationship to glenohumeral rotation in the shoulder of college baseball players. Am J Sports Med. 2002;30:354-360. PubMed ID: 12016075 doi:10.1177/03635465 020300030901

39. Myers JB, Laudner KG, Pasquale MR, Bradley JP, Lephart SM. Glenohumeral range of motion deficits and posterior shoulder tightness in throwers with pathologic internal impingement. Am J Sports Med. 2006;34:385-391. PubMed ID: 16303877 doi:10.1177/ 0363546505281804

40. Tehranzadeh AD, Fronek J, Resnick D. Posterior capsular fibrosis in professional baseball pitchers: case series of MR arthrographic findings in six patients with glenohumeral internal rotational deficit. Clin Imagin. 2007;31:343-348. doi:10.1016/j.clinimag.2007.05.005

41. Hodgins JL, Rubenstein W, Kovacevic D, Padaki A, Jobin CM, Ahmad CS. Pectoralis minor contracture in throwing shoulders of asymptomatic adolescent baseball players. Orthop J Sports Med. 2017;5:2325967117728041. PubMed ID: 28975133 doi:10.1177/ 2325967117728041

42. Jeon I-C. Correlation between the distance of scapular anterior tilting and the ratio of internal and external rotation angle of shoulder in supine position. J Phys Ther Sci. 2018;30:1329-1330. doi:10.1589/ jpts.30.1329

43. Borich MR, Bright JM, Lorello DJ, Cieminski CJ, Buisman T, Ludewig PM. Scapular angular positioning at end range internal rotation in cases of glenohumeral internal rotation deficit. J Orthop Sports Phys Ther. 2006;36:926-934. doi:10.2519/jospt.2006.2241 
44. Laudner KG, Moline MT, Meister K. The relationship between forward scapular posture and posterior shoulder tightness among baseball players. Am J Sports Med. 2010;38:2106-2112. doi:10. 1177/0363546510370291

45. Thomas SJ, Swanik KA, Swanik CB, Kelly JD. Internal rotation deficits affect scapular positioning in baseball players. Clin Orthop Relat Res. 2010;468:1551-1557. PubMed ID: 19841995 doi:10. 1007/s11999-009-1124-z

46. Mihata T, Jun BJ, Bui CNH, et al. Effect of scapular orientation on shoulder internal impingement in a cadaveric model of the cocking phase of throwing. J Bone Joint Surg Am. 2012;94A:1576-1583. doi:10.2106/JBJS.J.01972

47. Michener LA, McClure PW, Karduna AR. Anatomical and biomechanical mechanisms of subacromial impingement syndrome. Clin Biomech. 2003;18:369-379. doi:10.1016/S0268-0033(03) 00047-0

48. Seitz AL, McClure PW, Finucane S, Boardman ND, Michener LA. Mechanisms of rotator cuff tendinopathy: intrinsic, extrinsic, or both? Clin Biomech. 2011;26:1-12. doi:10.1016/j.clinbiomech.2010. 08.001
49. Leong HT, Tsui SS, Ng GY, Fu SN. Reduction of the subacromial space in athletes with and without rotator cuff tendinopathy and its association with the strength of scapular muscles. J Sci Med Sport. 2016;19:970-974. PubMed ID: 27102401 doi:10.1016/j.jsams.2016. 03.011

50. Bury J, West M, Chamorro-Moriana G, Littlewood C. Effectiveness of scapula-focused approaches in patients with rotator cuff related shoulder pain: a systematic review and meta-analysis. Man Ther. 2016;25:35-42. PubMed ID: 27422595 doi:10.1016/j.math.2016. 05.337

51. Saito H, Harrold ME, Cavalheri V, Mckenna L. Scapular focused interventions to improve shoulder pain and function in adults with subacromial pain: a systematic review and meta-analysis. Physiother Theory Pract. 2018;34:653-670. PubMed ID: 29351510 doi:10. 1080/09593985.2018.1423656

52. De Baets L, Jaspers E, Desloovere K, Van Deun S. A systematic review of 3D scapular kinematics and muscle activity during elevation in stroke subjects and controls. J Electromyogr Kinesiol. 2013;23:3-13. PubMed ID: 22841483 doi:10.1016/j.jelekin.2012. 06.007 